\title{
Editorial
}

\section{Cheating Death: what can we learn from tumour resistance?}

\author{
RA Knight*,1 \\ 1 Department of Cystic Fibrosis, National Heart and Lung Institute, Imperial \\ College School of Medicine, Emmanuel Kaye Building, Manresa Road, London \\ SW3 6LR, UK \\ * Corresponding author: RA Knight; E-mail: r.a.knight@imperial.ac.uk
}

Cell Death and Differentiation (2004) 11, S8-S9.

doi:10.1038/sj.cdd.4401463

Tumour cells and bacteria share a common property - they frequently acquire resistance to the therapeutics that are administered to treat them. Unfortunately, oncologists and microbiologists often contribute to the problem, as the drugs they use form part of the ongoing selection pressures that can facilitate resistance. In many ways, however, the task of the oncologist is more difficult than that of the microbiologist, as mammalian cells are inherently more complex than their bacterial counterparts. Tumour cells become resistant to therapy by a correspondingly wider range of mechanisms many of which prevent drug-induced cell death. Three papers and one review ${ }^{1-4}$ in this issue of CDD address the problem of tumour resistance to therapy and how we might circumvent this resistance to successfully treat patients with cancer, see Summary.

In their review, ${ }^{1}$ Reinhard Kofler and colleagues highlight the long-known observation that thymocytes from p53-null mice are resistant to apoptosis that is induced by radiation or chemotherapy, but remain susceptible to glucocorticoid (GC)induced apoptosis. ${ }^{5}$ As p53 is commonly mutated in haematological malignancies, GCs are an attractive therapeutic option. Indeed, when a number of prognostic factors were assessed (reviewed in Plasschaert et al. ${ }^{6}$ ), the long-term survival in patients with acute lymphoblastic leukaemia (ALL) was found to correlate with their response to an 8-day course of the GC prednisolone, even in patient subgroups with a predicted poor outcome. However, leukaemic cells from a minority of patients are insensitive to steroids such as GCs, so it is important to find additional therapies that circumvent this resistance.

So, what is the mechanism of GC-induced apoptosis, and so what could be the cause of resistance? Upon binding by $\mathrm{GC}$, the activated glucocorticoid receptor (GR) translocates to the nucleus and activates (or represses) the transcription of a large variety of target genes, some of which modulate cell death. Clearly, resistance to GCs could arise from abnormalities in the GR itself or in the complex downstream signalling pathways that are activated in response to GC binding. Two leukaemic cell lines - CEM and Jurkat - are commonly used for studies of GC resistance, and both have heterozygous mutations in the GR. In their original research paper, ${ }^{2}$ Kofler's group shows that the mutation in the Jurkat GR reduces its transcriptional activity, but not its binding to GC or its translocation to the nucleus. However, care must be taken in the application of data obtained using leukaemic cell lines to leukaemic cells in vivo. Although the GR mutation found in Jurkat cells has also been reported in a non-leukaemic patient with primary cortisol resistance, ${ }^{7}$ the GR mutation in CEM cells was only found in a minority of archival biopsies of the patient from whom the cell line was established. ${ }^{8}$ Therefore, GR mutations might not fully account for in vivo steroid resistance, though GR mutations could be selected in response to therapy.

Jurkat cells do not rely on just one mechanism for mediating resistance to steroids. In addition to the reduced transcriptional activity of the mutant GR in Jurkat cells, their low basal GR expression is not increased at either mRNA or protein levels by corticosteroid treatment, as occurs in steroidsensitive cells. Similar results were obtained in GC-resistant CEM sublines. ${ }^{2}$ By contrast, GR expression is increased following exposure to GC in GC-sensitive CEM sublines, and this autoinduction of GR (rather than the more usual repression) seems to be an important determinant of GC sensitivity. Although the mechanism of GR induction is not completely clear, the increased expression of GR mRNA in GC-sensitive leukaemia cells, which is seen by real-time, reverse-transcriptase (RT)-PCR, argues that at least one pathway is transcriptional. Further studies are needed to define whether activated GRs or other unrelated transcription factors are responsible, and why these enhance GR expression in some contexts and reduce it in others.

GCs have many metabolic actions; for example, in cultured rat hippocampal neurons, GCs reduce the total intracellular levels of glutathione - particularly its reduced form. ${ }^{9}$ The susceptibility of cells to apoptosis has been attributed, in part, to the cellular redox state, which is mainly determined by the ratio of oxidised and reduced forms of thiol groups on glutathione and proteins: the oxidised (-S-S-) forms favour apoptosis and the reduced $(-\mathrm{SH})$ forms inhibit cell death. ${ }^{10}$ Conceptually, therefore, by preferentially lowering levels of reduced glutathione, GC might promote apoptotic susceptibility, although this remains to be formally demonstrated. In support of this, a GC-resistant pre-B-cell leukaemic cell line has been shown to have higher glutathione levels than GCsensitive parental cells. ${ }^{11}$ Now, Klaus Debatin's group has extended these findings by showing that CEM cells that are resistant to a number of anticancer drugs also have a higher glutathione content, and reduction in glutathione biosynthesis is accompanied by restoration of drug sensitivity. ${ }^{3}$ The lowering of glutathione seems to affect both mitochondrial and death-receptor pathways of apoptosis induction. Resistance is a dominant phenotype, as hybrids of sensitive and resistant cells remain drug resistant, although again drug 
sensitivity of the fused cells could be restored by inhibiting the synthesis of glutathione.

TRAIL is one of the ligands - along with tumour necrosis factor $\alpha$ and CD95L (FasL) - that binds to death receptors on the cell surface. There are five TRAIL receptors (reviewed in ${ }^{12}$ ), but only two - DR4 and DR5 - can activate the apoptotic programme after ligation. Functional death receptors contain a cytoplasmic death domain, and oligomerisation of DR4 and DR5 following TRAIL binding results in recruitment of adaptor proteins to the death domain to form the Death-Inducing Signalling Complex (DISC). Procaspase-8 interacts with the DISC, where it is proteolytically cleaved to produce the active enzyme that initiates the caspase cascade and results in apoptosis.

Tumour Necrosis Factor (TNF)- $\alpha$-related Apoptosis-inducing Ligand (TRAIL) is an attractive therapeutic prospect in cancer for two main reasons. First, TRAIL receptor signalling, like GCinduced death, is independent of $\mathrm{p} 53$, and therefore remains effective in tumours in which $p 53$ is mutated. Second, a number of studies have already shown that, in general, TRAIL selectively induces apoptosis in malignant cells, but not in normal cells. However, not all tumours are sensitive to TRAIL, and Henning Walczak, Peter Krammer and their colleagues have investigated the mechanisms of TRAIL resistance, and how they may be overcome, in hepatocellular carcinoma (HCC) cell lines. ${ }^{4}$ In vivo, $\mathrm{HCC}$ is often chemoresistant; however, as has been described for some other tumours, TRAIL-mediated killing of resistant HCC lines can be restored by treatment with 5 -fluorouracil (5-FU), and it is the mechanism by which this occurs that the paper is principally concerned with. The authors identify three main actions of 5-FU, including upregulation of active TRAIL receptors, enhanced recruitment of proteins to the DISC and reduced expression of FLIP - a caspase-like protein that lacks a catalytic domain and that inhibits caspase- 8 activation at the DISC. Of these, the increase in DISC formation and caspase-8 activation seem to be the most important, as the levels of FLIP in the DISCs of TRAIL-sensitive and -resistant lines are similar. Reduction of FLIP by RNA interference does, however, restore sensitivity to resistant cells, and this may be a therapeutic option worth pursuing. The effectiveness of combining conventional chemotherapy and TRAIL therapy in cells that are otherwise resistant to each agent alone is exciting, although, on the 'first, do no harm' principle, it needs to be demonstrated that the combination therapy is specific to malignant cells.

Like the microbiologist fighting antibiotic resistance, the oncologist and the cancer cell are similarly locked in an arms race. As more and more sophisticated anticancer strategies are developed, so the malignant cell reveals ever more subtle ways of evading them. Alas, there is, as yet, no sign that the evasive potential of cancer cells is approaching exhaustion, and the cold war between malignancy and its management looks set to continue escalating. But there may be a positive fall out from this conflict. In cancer, we have to understand why the aberrant cell does not do the decent thing and invoke the default option of apoptosis. In other clinical contexts, such as neurodegeneration and AIDS, however, it is the death processes themselves that are excessive. Understanding how cancer cells cheat death could reveal mechanisms that we can target therapeutically to salvage other cells from inappropriate suicide.

\section{Key points}

- Resistance to apoptosis can cause resistance to anticancer therapeutics.

- Mutation of the glucocorticoid receptor (GR) and failure of GR autoinduction in response to glucocorticoid (GC) resulting in the inability of GC to induce apoptosis in lymphoid malignancies.

- High intracellular levels of the antioxidant glutathione (GSH), particularly of the reduced form, are also associated with chemoresistance in malignant lymphoid cells.

- The failure of Tumour Necrosis Factor (TNF)- $\alpha$-related Apoptosis-inducing Ligand (TRAIL) to cause cell death in hepatocellular carcinoma cell lines is associated with impaired recruitment to TRAIL receptors of the accessory molecules necessary for activation of the apical caspase, caspase-8.

1. Riml S et al. (2004) Cell Death Differ. 11(S1): S65-S72

2. Schmidt $S$ et al. (2004) Cell Death Differ. 11(S1): S45-S55

3. Friesen $C$ et al. (2004) Cell Death Differ. 11(S1): S73-S85

4. Ganten TM et al. (2004) Cell Death Differ. 11(S1): S86-S96

5. Clark AR et al. (1993) Nature 362: 849

6. Plasschaert SLA et al. (2004) Cancer Treatment Rev. 30: 37

7. Ruiz M et al. (2001) Clin. Endocrinol. 55: 363

8. Hillman AG et al. (2000) Cancer Res. 60: 2056

9. Patel $R$ et al. (2002) J. Neurochem. 82: 118

10. Marchetti $P$ et al. (1997) Eur. J. Immunol. 27: 289

11. Inoue $\mathrm{H}$ et al. (2002) Jpn. J. Cancer Res. 93: 582

12. Wang $S$ and El-Deiry WS (2003) Oncogene 22: 8628 